\title{
Dynamic components of linear stable mixtures from fractional low order moments
}

\author{
Fabricius, Thomas; Kidmose, Preben; Hansen, Lars Kai
}

Published in:

Proceedings of International Conference on Acoustics, Speech and Signal Processing, ICASSP2001

Link to article, DOI:

10.1109/ICASSP.2001.940710

Publication date:

2001

Document Version

Publisher's PDF, also known as Version of record

Link back to DTU Orbit

Citation (APA):

Fabricius, T., Kidmose, P., \& Hansen, L. K. (2001). Dynamic components of linear stable mixtures from fractional low order moments. In Proceedings of International Conference on Acoustics, Speech and Signal Processing, ICASSP2001 (Vol. 6, pp. 3957-3960). IEEE Press. https://doi.org/10.1109/ICASSP.2001.940710

\section{General rights}

Copyright and moral rights for the publications made accessible in the public portal are retained by the authors and/or other copyright owners and it is a condition of accessing publications that users recognise and abide by the legal requirements associated with these rights.

- Users may download and print one copy of any publication from the public portal for the purpose of private study or research.

- You may not further distribute the material or use it for any profit-making activity or commercial gain

- You may freely distribute the URL identifying the publication in the public portal 


\title{
DYNAMIC COMPONENTS OF LINEAR STABLE MIXTURES FROM FRACTIONAL LOW ORDER MOMENTS
}

\author{
Thomas Fabricius, Preben Kidmose and Lars Kai Hansen \\ Technical University of Denmark \\ Department for Mathematical Modelling \\ Richard Petersens Plads bldg. 321 \\ 2800 Lyngby \\ Denmark
}

\begin{abstract}
The second moment based independent component analysis scheme of Molgedey and Schuster is generalized to fractional low order moments, relevant for linear mixtures of heavy tail stable processes. The Molgedey and Schuster algorithm stands out by allowing explicitly construction of the independent components. Surpricingly, this turns out to be possible also for decorrelation based on fractional low order moments.
\end{abstract}

Keywords: Blind Source Separation (BSS), Dynamic Components, Independent Component Analysis (ICA), Stable process, Fractional lower order moments, Cocktail party problem

\section{INTRODUCTION}

Reconstruction of independent components from linear mixtures is an important signal processing research area with numerous practical applications $[1,2]$. Typically, independent component analysis (ICA) proceeds from non-linear transformations $[3,4]$ or from temporal correlations $[5,6]$. The second moment based Molgedey-Schuster approach assumes that the independent sources have different autocorrelation functions $[5,7,8]$. The main virtue of the approach is that an explicit solution (reconstruction of sources and mixing matrix) is possible. In [8] we applied the MolgedeySchuster algorithm to image mixtures and we proposed a minor modification of the algorithm that relieves a problem of the original approach, namely that it occasionally produces complex mixing coefficients and source signals. Attias and Schreiner proposed a very rich ICA framework based on higher order statistics and decorrelation $[9,10]$, allowing for completely general and learnable source distri-

This work is funded by the Danish Research Councils through the THOR Center for Neuroinformatics. butions, however at the price of significant additional computation.

In this contribution we analyze dynamic decorrelation for heavy tail stable random signal, and we provide a generalization of the Molgedey-Schuster algorithm based on fractional low order moments rather than second moments.

\section{STABLE DISTRIBUTIONS AND SOME PROPERTIES}

Random vectors following a stable distribution are stable with respect to addition, i.e., if $\mathbf{X}$ and $\mathbf{Y}$, are stable then so is the $\operatorname{sum} \mathbf{Z}=\mathbf{X}+\mathbf{Y}$. A random vector follows a strictly symmetric stable distribution iff its characteristic function has the form [11]

$$
\phi(\mathbf{t})= \begin{cases}\exp \left\{\mathbf{t}^{T} \mathbf{A t}\right\} & \alpha=2 \\ \exp \left\{\int_{S}\left|\mathbf{t}^{T} \mathbf{s}\right|^{\alpha} \mu(d S)\right\} & 0<\alpha<2\end{cases}
$$

where $\mathrm{t} \in R^{n}$ and $\mu(d S)$ is a finite Borel measure called the spectral measure, $S$ is the unit sphere and $\alpha$ is the characteristic exponent. A random variable $X$ that is distributed by a symmetric stable distribution with characteristic exponent $\alpha$ is denoted $X \sim S \alpha S$. In the class of stable distributions we find the normal distribution $\alpha=2$ and the so-called Cauchy distribution $\alpha=1$. In general the low order stable distributions have heavy tails, and are supposed to model, e.g., speech signals. Because of the heavy tails, higher order moments do not exists, hence, ICA methods based on second moments are not well-defined for these signals. For multivariate stable variables the spectral measure carries information about the dependencies among the individual components and this may be used for independent component analysis [12]. Here we show that independent dynamic components can be reconstructed explicitly a la Molgedey Schuster, even if the signals follow stable distributions. 
Our derivation is based on the generalized covariation between random variables $X$ and $Y$ jointly $S \alpha S$ as defined in [11]

$$
[X, Y]_{\alpha}=\int_{S} x y^{\langle\alpha-1\rangle} \mu(d S)
$$

with the definition $z^{\langle\alpha-1\rangle}=|z|^{\alpha-1} \operatorname{sign}(z)$. Note that $[X, Y]_{\alpha}=0$ is necessary but not sufficient for $X$ and $Y$ to be independent. The covariation has a convenient pseudolinearity property. Let $X_{1}, X_{2}, Y_{1}$ and $Y_{2}$ be jointly $S \alpha S$ and $Y_{1}, Y_{2}$ independent then

$$
\begin{aligned}
& {\left[a_{1} X_{1}+a_{2} X_{2}, b_{1} Y_{1}+b_{2} Y_{2}\right]_{\alpha}=} \\
& a_{1}\left[X_{1}, Y_{1}\right]_{\alpha} b_{1}^{\alpha-1}+ a_{2}\left[X_{2}, Y_{1}\right]_{\alpha} b_{1}^{\alpha-1}+ \\
& a_{1}\left[X_{1}, Y_{2}\right]_{\alpha} b_{2}^{\alpha-1}+a_{2}\left[X_{2}, Y_{2}\right]_{\alpha} b_{2}^{\alpha-1}
\end{aligned}
$$

From the covariation we can derive the covariation coefficient

$$
\lambda_{X, Y}=\frac{[X, Y]_{\alpha}}{[Y, Y]_{\alpha}}
$$

which has the property [11]

$$
\lambda_{X, Y}=\frac{E\left(X Y^{\langle p-1\rangle}\right)}{E\left(Y Y^{\langle p-1\rangle}\right)}
$$

for $1 \leq p<\alpha$. By equation (5) we can estimate the covariation coefficient from data.

\section{DECORRELATION BASED ON FRACTIONAL LOW ORIDER MOMENTS}

We are now equipped to generalize the Molgedey-Schuster approach, and start out by defining the matrix of observed signals $\mathbf{X}$ with elements

$$
X_{m, t}=\sum_{j=1}^{N_{s}} A_{m, j} S_{j, t} \quad 0 \leq t<T
$$

where $A_{m, j}$ are the mixing coefficients forming the mixing matrix $\mathbf{A}, S_{j, t}$ is an instance of the $j^{\text {th }} S \alpha S$ source signal written as an element in the matrix $\mathbf{S}$ and $N_{s}$ is the number of sources. We now assume that the different source signals are independent,

$$
\left[S_{j, t}, S_{i, t+\tau}\right]_{\alpha}=0 \quad j \neq i \quad \tau \in R .
$$

The covariation coefficient between $X_{m, t}$ and $X_{n, t+\tau}$ can be found using equations (4) and (6)

$$
\lambda_{X_{m, t}, X_{n, t+\tau}}=\frac{\left[\sum_{j=1}^{N_{s}} A_{m, j} S_{j, t}, \sum_{i=1}^{N_{s}} A_{n, i} S_{i, t+\tau}\right]_{\alpha}}{\left[\sum_{i=1}^{N_{s}} A_{n, i} S_{i, t+\tau}, \sum_{i=1}^{N_{s}} A_{n, i} S_{i, t+\tau}\right]_{\alpha}}
$$

Finally, we use that the sources are independent, hence, equation (3) is valid, providing

$$
\lambda_{X_{m, t}, X_{n, t+\tau}}=\frac{\sum_{j=1}^{N_{s}} \sum_{i=1}^{N_{s}} A_{m, j}\left[S_{j, t}, S_{i, t+\tau}\right]_{\alpha} A_{n, i}^{\langle\alpha-1\rangle}}{\sum_{j=1}^{N_{s}} \sum_{i=1}^{N_{s}} A_{n, j}\left[S_{j, t+\tau}, S_{i, t+\tau}\right]_{\alpha} A_{n, i}^{\langle\alpha-1\rangle}}
$$

We define matrices $\Lambda^{(\tau)}$ with elements $\Lambda_{m n, n}^{(\tau)}=\lambda_{X_{m, t}, X_{n, t+\tau}}$, $\mathbf{B}^{(\tau)}$ with the nominator of equation (8) as elements

$$
B_{m, n}^{(\tau)}=\sum_{j=1}^{N_{s}} \sum_{i=1}^{N_{s}} A_{m, j}\left[S_{j, t}, S_{i, t+\tau}\right]_{\alpha} A_{n, i}^{\langle\alpha-1\rangle}
$$

and a diagonal matrix $\mathbf{D}$ with the denominator from equation (8) as elements

$$
D_{n, n}=\sum_{j=1}^{N_{s}} \sum_{i=1}^{N_{s}} A_{n, j}\left[S_{j, t+\tau}, S_{i, t+\tau}\right]_{\alpha} A_{n, i}^{\langle\alpha-1\rangle}
$$

Assuming stationarity, $\mathbf{D}$ is independent of $\tau$, so that $\mathbf{\Lambda}^{(\tau)}=$ $\mathbf{B}^{(\tau)} \mathbf{D}^{-1}$. From equation (10) it is seen that $\mathbf{B}^{(\tau)}$ decomposes into

$$
\mathbf{B}^{(\tau)}=\mathbf{A} \mathbf{C}^{(\tau)} \mathbf{A}^{T^{\langle\alpha-1\rangle}}
$$

where $\mathbf{A}^{T^{\langle\alpha-1\rangle}}$ is the signed norm applied to each element of $\mathbf{A}^{T} . \mathbf{C}^{(\tau)}$ is the matrix with elements $C_{j, i}^{(\tau)}=$ $\left[S_{j, t}, S_{i, t+\tau}\right]_{\alpha}$, and we notice that $\mathbf{C}^{(\tau)}$ is diagonal since the sources are independent. We next form the quotient matrix $\mathbf{Q}^{(\tau)}$

$$
\begin{aligned}
\mathbf{Q}^{(\tau)} & =\mathbf{\Lambda}^{(\tau)} \mathbf{\Lambda}^{(0)^{-1}}=\mathbf{B}^{(\tau)} \mathbf{B}^{(0)-1} \\
& =\mathbf{A} \mathbf{C}^{(\tau)} \mathbf{A}^{T^{\langle\alpha-1\rangle}} \mathbf{A}^{\left.T^{\langle\alpha-1}\right)^{-1}} \mathbf{C}^{(0)^{-1}} \mathbf{A}^{-1} \\
& =\mathbf{A} \mathbf{C}^{(\tau)} \mathbf{C}^{(0)^{-1}} \mathbf{A}^{-1}
\end{aligned}
$$

We can now construct the mixing matrix $\mathbf{A}$ by solving the eigenvalue problem,

$$
\mathbf{Q}^{(\tau)} \mathbf{\Psi}=\mathbf{\Psi} \boldsymbol{\Gamma}
$$

if we identify $\boldsymbol{\Psi}=\mathbf{A}$ and $\left.\mathbf{\Gamma}=\mathbf{C}^{(\tau)} \mathbf{C}^{(0)}\right)^{-1}$.

In short, we have shown our main result, namely that it is possible to recover the mixing matrix from fractional low order moments. The source signals are subsequently estimated by $\mathbf{S}=\mathbf{A}^{-1} \mathbf{X}$.

\subsection{Sample estimates of Fractional Low Order Moments}

Equation (5) provides the estimate of the elements of $\Lambda^{(\tau)}$

$$
\hat{\lambda}_{X_{m, t}, X_{n, t+\tau}}=\frac{\frac{1}{T-\tau} \sum_{t=0}^{T-1-\tau} X_{m, t} X_{n, t+\tau}^{\langle p-1\rangle}}{\frac{1}{T} \sum_{t=0}^{T-1} X_{n, t} X_{n, t}^{\langle p-1\rangle}}
$$


from a sample of $T$ measurements. We can then form

$$
\begin{aligned}
\widehat{\mathbf{Q}}^{(\tau)} & =\widehat{\boldsymbol{\Lambda}}^{(\tau)} \hat{\boldsymbol{\Lambda}}^{(0)-1} \\
& =\frac{T}{T-\tau} \mathbf{X X}^{(\tau)} \mathbf{X}^{\langle p-1\rangle^{T}}\left(\mathbf{X} \mathbf{X}^{\langle p-1\rangle^{T}}\right)^{-1}
\end{aligned}
$$

hence, solve the eigenvalue problem to obtain the estimated mixing matrix. In the above equation $\mathbf{X}^{(\tau)}$ is the zero padded matrix $\mathbf{X}$ shifted by a lag $\tau$. The choice of $\tau$ is a question one have to deal with. The optimal choice will both depend on the accuracy on the estimated elements in $\mathbf{C}^{(\tau)}$ and $\mathbf{C}^{(0)}$ and on the actual true values in $\mathbf{C}^{(\tau)} \mathbf{C}^{(0)^{-1}}$. $\tau$ should be selected so we have the largest difference in the eigenvalues of $\mathbf{Q}^{(\tau)}$ i.e. in $\mathbf{C}^{(\tau)} \mathbf{C}^{(0)^{-1}}$ compared to their accuracy. In this paper we will not address this further, but suggest using $\tau=1$.

\section{EXPERIMENTAL RESULTS}

We illustrate the performance of the generalization of the Molgedey-Schuster algorithm by analyzing mixing of synthetic data following a stable distribution, and by mixing of two speech signals.

\subsection{Synthetic data}

We perform experiments on mixing of two signals in two channels. In the first we mix two independent unit-variance Gaussian signals by the mixing matrix

$$
\mathbf{A}=\left[\begin{array}{ll}
0.8944 & 0.4472 \\
0.3714 & 0.9285
\end{array}\right]
$$

containing two non-orthogonal normalized rows. The source signals have different temporal dependencies, one is white noise, while the other is low-pass filtered with a FIR-filter with two coefficients $h_{0}=1$ and $h_{1}=0.8$. We show in Figure 1 scatterplots of the "true signals" and the recovered signals from the estimated mixing matrix estimated from the conventional Molgedey-Schuster algorithm. Next we mix two similar stable signals drawn from distributions with $\alpha=1.5$, hence with heavy tails, by the same mixing matrix. In Figure 2 we show the scatterplots of the true and estimated signals.

The two different scenarios lead to similar estimated mixing matrices

$$
\mathbf{A}=\left[\begin{array}{ll}
0.9034 & 0.4287 \\
0.2812 & 0.9597
\end{array}\right] ; \quad \mathbf{A}=\left[\begin{array}{ll}
0.9367 & 0.3503 \\
0.4272 & 0.9041
\end{array}\right]
$$

demonstrating that it is possible reliably to reconstruct independent stable source signals by the generalized MolgedeySchuster scheme, in cases where the second moment correlations would not converge for large samples.

\subsection{Speech data}

Finally, we consider the blind separation of a mixture of two speech signals. The signals are two source signals used in [13]. We use the same mixing matrix as in the above example. In Figure 3 we show the scatterplot of the true and estimated signals using the conventional Molgedey-Schuster algorithm and in Figure 4 using the new scheme using $p=$ 1.8. The recovered signals are very similar, indicating that the conventional algorithm is robust to the underlying distribution of the source signals. We conjecture that this robustness is related to the fact that the conventional MolgedeySchuster algorithm proceeds from the ratio of two second order moments, and that the ratio might have better statistical properties than the two individual moments, this is the topic for on-going research.

\section{CONCLUSION}

We have derived a new algorithm generalizing that of Molgedey and Schuster [5] based on fractional low order moments. We have provided a scheme for estimating the relevant moments from finite samples and demonstrated the viability of the new algorithm in a synthetic data set. However, we also found that the original algorithm is fairly robust to possibly diverging second order moments, and conjectured that the explanation could be related to the fact that the conventional algorithm is based on the ratio between two diverging terms, possibly improving the statistical properties.
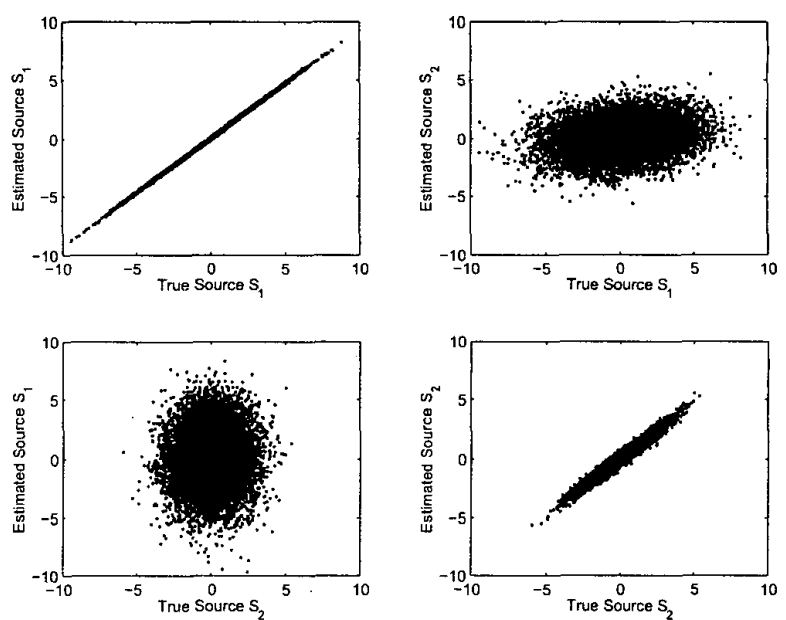

Fig. 1. Original Gaussian source, i.e. $\alpha=2$ signals against recovered source signals when using the original Molgedey and Schuster algorithm $(p=2)$. In this setup $T=10000$ and one of the sources was white, the other filtered by the FIR-filter in the text. The algorithm was run at $\tau=1$ 

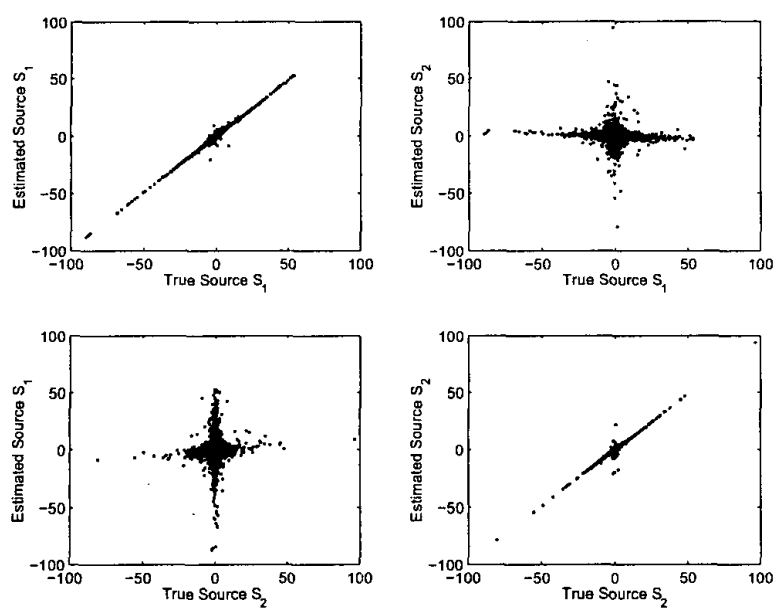

Fig. 2. Same setup as in Figure 1 but now the source signals are generated with $\alpha=1.5$ and the algorithm is run with $p=1.49$.
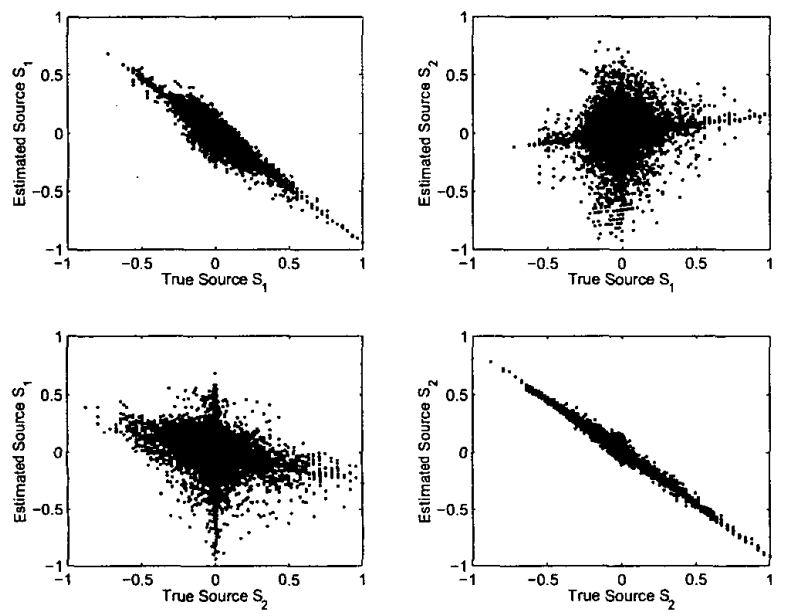

Fig. 3. Original speech source signals against recovered source signals when using the original Molgedey and Schuster algorithm. The speech signals had a duration of $T=23000$. The algorithm was run at $\tau=5$.

\section{REFERENCES}

[1] T.-W. Lee, Independent Component Analysis: Theory and Applications, Kluwer Academic Publishers, New York, 1998.

[2] M. Girolami (Ed.), Advances in Independent Component Analysis., Springer-Verlag, New York, 2000.

[3] P. Comon, "Independent component analysis: A new concept?," Signal Processing, vol. 36, pp. 287-314, 1994.

[4] A. Bell and T.J. Sejnowski, "An information-maximization
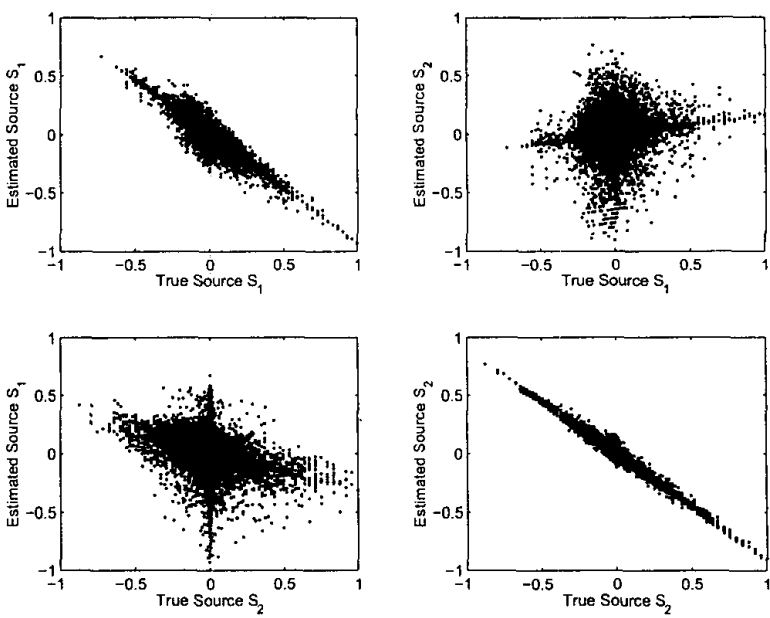

Fig. 4. The setup is the same as that in Figure 3 but with $p=1.8$

approach to blind separation and blind deconvolution," $\mathrm{Neu}$ ral Computation, vol. 7, pp. 1129-1159, 1995.

[5] L. Molgedey and H. Schuster, "Separation of independent signals using time-delayed correlations," Physical Review Letters, vol. 72, no. 23, pp. 3634-3637, 1994.

[6] A. Belouchrani, K. Abed-Meraim, J.-F. Cardoso, and E. Moulines, "Blind source separation technique using second-order statistics," IEEE Transactions on Signal Processing, vol. 45, no. 2, pp. 434-444, 1997.

[7] L.K. Hansen and J. Larsen, "Source separation in short image sequences using delayed correlation," Proceedings of the IEEE Nordic Signal Processing Symposium, Vigsø, Denmark 1998. Eds. P. Dalsgaard and S.H. Jensen, pp. 253-256, 1998.

[8] L.K. Hansen, J. Larsen, and T. Kolenda, "On independent component analysis for multimedia signals," L. Guan, S.Y. Kung and J. Larsen (eds.) Multimedia Image and Video Processing, CRC Press, 2000.

[9] H. Attias and C.E. Schreiner, "Blind source separation and deconvolution by dynamic component analysis," Neural Networks for Signal Processing VII: Proceedings of the 1997 IEEE Workshop, 456-465 (1997, pp. 456-465, 1997.

[10] H. Attias and C.E. Schreiner, "Blind source separation and deconvolution: The dynamic component analysis algorithm," Neural Computation, vol. 10, pp. 1373-1424, 1998.

[11] C. L. Nikias and M. Shao, Signal Processing with AlphaStable Distributions and Application., Wiley, New York, 1995.

[12] P. Kidmose, "Independent components of linear stable mixtures," Preprint, submitted to ICASSP'200I, 2000.

[13] T-W. Lee, M.S. Lewicki, M. Girolami, and T.J. Sejnowski, "Blind source separation of more sources than mixtures using overcomplete representations," IEEE Signal Processing Letters, vol. 6, no. 4, 1999. 